\title{
Über die Entstehung der solaren Komponente der Ultrastrahlung
}

\author{
Von Ludwig Biermann und Erich Bagge \\ Aus dem Max-Planck-Institut für Physik, Göttingen, \\ und dem Physikalischen Staatsinstitut, Hamburg \\ (Z. Naturforschg. 4 a, 303-315 [1949]; eingegangen am 24. Dezember 1948)
}

Die Beobachtungen von $\mathrm{F}$ orbush und $\mathrm{Ehmert}$ machen es sehr wahrscheinlich, daß gelegentlich Ultrastrahlung von der Sonne emittiert wird und daß hierfür Vorgänge in der Umgebung aktiver Sonnenflecken verantwortlich sind.

Die Änderungen der Magnetfelder aktiver Flecken ergeben, wie schon $\mathrm{S}$ wa $\mathrm{n}$ n bemerkt hat, induzierte elektrische Felder, deren Ringintegrale Werte bis zu $10^{9-10^{10} \mathrm{~V}}$ annehmen. Bisher war jedoch nicht zu verstehen, auf welchem Wege geladene Teilchen in diesen Feldern die diesen Potentialdifferenzen entsprechenden Energien erlangen können.

Es wird zunächst ein sehr spezieller Modellmechanismus betrachtet, bei dem die Bewegungsgleichung geschlossen integriert werden kann. Dann werden die Bewegungsverhältnisse allgemein diskutiert, und es wird gezeigt, daß gerade in komplizierteren aktiven Fleckengruppen Beschleunigungen $\| \mathfrak{S}$ auftreten werden, welche auf Ultrastrahlenergie führen können; wenn dies auf magnetischen Feldlinien geschieht, die in große Entfernung vom Fleck führen, können die beschleunigten Teilchen die Sonne verlassen.

Diese Diskussion gilt zunächst nur für sich ändernde Magnetfelder in derart verdünnter Materie, daß ihre Rückwirkung auf die Ausbreitung der Magnetfelder vernachlässigt werden darf. Tatsächlich erweist sich die Frage dieser Rückwirkung der Sonnenatmosphäre als' sehr verwickelt. Andererseits ist es für die Entstehung der Ultrastrahlung hinreichend, wenn im Koronaniveau die Magnetfelder nur (größenordnungsmäßig) ihre Vakuumintensität haben und hinsichtlich der Regellosigkeit ihrer Gestalt und Veränderungen den Erscheinungen im Niveau der'sichtbaren Oberfläche entsprechen; die ausführliche Diskussion erweist diese Vorstellung als begründet.

Schließlich werden die energetischen Verhältnisse diskutiert; es wird gezeigt, daß die beobachteten Intensitäten der Ultrastrahlungsspitzen verstanden werden können. Falls die hier ausgeführten Vorstellungen zutreffen, ist zu erwarten, daß das Energiespektrum der solaren Ultrastrahung keine untere Grenze bei $10^{9}$ Volt besitzt, sondern daß viel mehr Energie durch weniger energiereiche Teilchen transportiert wird. Ferner zeigt sich, daß eine lokale Erniedrigung der Teilchendichtè wesentlich für die Entstehung von Ultrastrahlung ist.

Im letzten Abschnitt wird die Frage diskutiert, ob die gesamte Ultrastrahlung durch Mechanismen der in dieser Arbeit vorgeschlagenen Art entstanden sein und entstehen könnte: in diesem Zusammenhang wird der Einfluß der Expansion der Welt auf das Energiespektrum der Ultrastrahlung diskutiert.

$\mathrm{E}$ s gibt heute bereits eine Reihe von unabhängigen Hinweisen dafür, daß auf der Sonne gelegentlich aktive Bereiche vorhanden sind, die kosmische Strahlung emittieren und so Intensitätsschwankungen dieser Strahlung auf der Erde hervorrufen können. Hierauf haben neuerdings $\mathrm{Forbush}{ }^{1}$ und $\mathrm{Ehmer \textrm {m } ^ { 2 }}$ hingewiesen, welche deutliche Zusammenhänge zwischen dem Auftreten von chromosphärischen Eruptionen und Intensitätsspitzen der Höhenstrahlung be-

1 S. E. F o r bu sh, Physic. Rev. 70, 771 [1946].

2 A. Ehmert, Vortrag auf d. Physikertagung Göttingen, Sept. 1947. Vgl. Z. Naturforschg. 3 a, 264 [1948]. obachteten. Diese Feststellungen wurden, z. Tl. jedenfalls, durch Messungen anderer Autoren ${ }^{3}$ bestätigt, so daß Zweifel an der Realität des Phänomens kaum mehr möglich sind.

Andererseits hat $\mathrm{He} B^{4}$ schon im Jahre 1933 bemerkt, daß man den mit der Ortszeit des jeweiligen Beobachters veränderlichen Tagesgang dieser Strahlung mit seinem Teilchenmaximum in den frühen Nachmittagsstunden am einfachsten

3 A. D u p e r i e r, Proc. physic. Soc. 57, 473 [1947]; D. W. N. D o l b e a r u. H. Ell i ot, Nature [London] 159, 58 [1947]; H. V. N e h e r u. W. C. R o es'ch, Rev. mod. Physics 20, 350 [1948].

4 V. F. H e ß, Nature [London] 159, 58 [1947]. 
mit einer Eigenproduktion von Ultrastrahlung durch die Sonne erklären könne. Darüber hinausgehend weist D u p e ri e ${ }^{5}$, der seinen Registrierungen ein deutliches Minimum der Amplitude des Tagesganges in den Wintermonaten entnimmt, darauf hin, daß dieser Effekt als Folge der veränderlichen Zenithdistanz der Sonne im Laufe des Jahres zu verstehen ist. Es ist dabei möglich, diese Erscheinungen als Folge einer mäßigen Anzahl kleiner Intensitätsspitzen aufzufassen.

Schließlich aber kann die 27-Tage-Periode der kosmischen Strahlung, die nach den Untersuchungen von $\mathrm{Brox}$ on ${ }^{6}$ eine sehr enge positive Korrelation zur relativen Größe der Fleckengebiete und deren durch die Sonnenrotation erzeugten 27-Tagesgang besitzt, in der Eigenproduktion von Höhenstrahlung durch die Sonne ihre einfache und natürliche Erklärung finden.

In Anbetracht dieser Sachlage scheint es uns berechtigt, die Frage nach dem Mechanismus aufzuwerfen, der den Ultrastrahlungspartikeln auf der Sonne die erforderlichen hohen Energien der Größenordnung $10^{9}-10^{10} \mathrm{eV}$ verleiht.

Wenn Ultrastrahlung von der Sonne zur Erde gelangt, so ist dies nur möglich, sofern kein allgemeines Magnetfeld der Sonne der von $\mathrm{Hale}$ postulierten Art besteht; hierauf hat schon F o r bush ${ }^{1}$ hingewiesen. Die Beobachtungsbefunde über dieses Feld sind bekanntlich sehr widerspruchsvoll; daß es mindestens zeitweise unmeßbar klein ist, scheint schon sicher zu sein. Wir werden es daher im folgenden außer Betracht lassen.

Von Sw a n ${ }^{7}$ wurde schon im Jahre 1933 ein Mechanismus beschrieben, der es erlauben sollte, Ladungsträger in elektrischen Ringfeldern um die sich ändernden Magnetfelder der Sonnenflecken zu beschleunigen. Bei der Diskussion dieses Vorganges ergibt sich aber die schon von Swann selbst besprochene Schwierigkeit, daß dieser Beschleunigungsmechanismus im Grunde nur im ersten Augenblick des eben beginnenden Aufbaus eines Fleckens ablaufen kann. Es ist nämlich erforderlich, daß zwar $\dot{H}$ sehr groß, $H$ selbst aber noch verschwindend klein $\left(H \approx 10^{-3} \mathrm{G}\right)$ ist.

${ }^{5}$ A. D u p e r i e r, Nature [London] 158, 196 u. 974 [1946].

${ }^{6}$ F. W. B r ox o n, Physic. Rev. 62, 507 [1942].

7 W. F. G. S w a n n, Physic. Rev. 43, 217 [1933].

8 E. B a g g e u. L. B i e r m a n n, Naturwiss. 1948.

9 S. dazu C h a p m a n, Monthly Notices Roy. astronom. Soc. 103, 117 [1913], u. F. W. B r ox on ${ }^{6}$.
Nur dann nämlich werden die vereinigten Wirkungen des elektrischen und des magnetischen Feldes auf die Teilchen die nötigen Energien der Größenordnung $10^{9}-10^{10} \mathrm{eV}$ übertragen.

In einer früheren Note ${ }^{8}$ haben wir schon darauf hingewiesen, daß es jedoch andere Möglichkeiten gibt, auf Ladungsträger größere Energien zu übertragen, ohne daß hier die Swannschen Schwierigkeiten auftreten, und wir wollen im folgenden verschiedene Modelle im einzelnen diskutieren.

\section{Die Vorgänge in Sonnenflecken- gruppen}

Es ist bekannt, daß sich der Verlauf der magnetischen Kraftlinien in Fleckenfeldern in einiger Näherung durch eine geeignete Verteilung magnetischer Dipole darstellen läßt. Die entsprechenden Dipolmomente erreichen ${ }^{*}$ in Extremfällen Werte der Größenordnung einiger $10^{31} \mathrm{Gau} ß \mathrm{~cm}^{3}$. Außerdem wird beobachtet, daß bei Fleckengruppen oft Zentren verschiedener Polarität nahe beieinander liegen und diese rasche Bewegungen ausführen, indem sie sich gegenseitig annähern oder auseinanderstreben ${ }^{10}$. Häufig treten auch neben zeitlich langsam veränderlichen Flecken plötzlich neue Flecken anderer oder gleicher Polarität auf und verschwinden dann wieder. In allen diesen Fällen müssen die sich ändernden magnetischen Felder auch elektrische Wirbelfelder induzieren, die geladene Teilchen beschleunigen können, und es erhebt sich nur die Frage, ob es unter den vielen Möglichkeiten für die räumliche Verteilung der elektrischen und magnetischen Felder auch solche gibt, die einerseits den Teilchen Energien der Größenordnung $10^{9}-10^{10} \mathrm{eV}$ übermitteln und die andererseits auch so gerichtet sind, daß die Partikel die Sonne verlassen können. Um diese Frage entscheiden zu können, idealisieren wir die Vorgänge in den Fleckengruppen in bestimmter Weise und diskutieren die dort auftretenden Möglichkeiten der Teilchenbewegungen. Hierbei nehmen wir an, daß die Fleckfelder sich in der Korona etwa

10 Welche hydrodynamischen Vorgänge diese Bewegungen hervorrufen und welcher Art die elektromotorischen Kräfte sind, die letzten Endes die Magnetfelder erzeugen, soll hier nicht diskutiert werden. T. G. C o w ling hat gezeigt, daß die Magnetfelder der Flecken nicht im Photosphärenniveau primär erzeugt werden können, und daß in diesem Niveau Bewegungen quer zu den magnetischen Feldlinien sehr stark behindert werden. Vgl. Monthly Notices Roy. astronom. Soc. 94, 39 [1934]. 
wie im Vakuum ausbreiten. Diese Annahme werden wir noch näher diskutieren; hier bemerken wir nur, daß sie im Einklang ist mit den Schlüssen, die sich aus den Beobachtungen an Protuberanzen in derNähe von Flecken ziehen lassen.

Die Teilchenbewegung in sich ändernden bipolaren. Fleckenpaaren

Es werde angenommen, daß zwei magnetische Dipole der Momente $\mu_{1}$ und $\mu_{2}$, die die zwei Sonnenflecken repräsentieren sollen, im Abstand $2 D$ voneinander sich mit der Geschwindigkeit $v$ aufeinander zubewegen. Ihre Achsen seien senkrecht zur Sonnenoberfläche gerichtet; der Dipol B

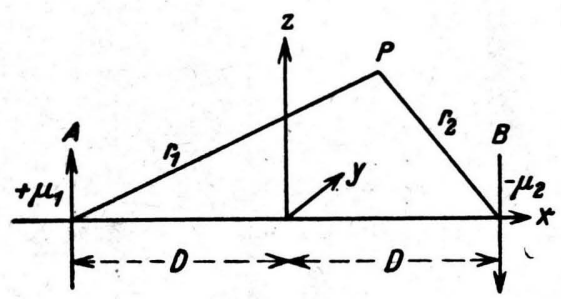

Abb. 1. Lage der Dipole.

(Abb.1) bewege sich mit der Geschwindigkeit $v$ auf A zu $\left(v / c \approx 10^{-6}\right)$. Dann hat das Potential $V$ der beiden Dipolfelder im Punkte P den Wert:

$$
V=\frac{\mu_{1} z}{r_{1}^{3}}-\frac{\mu_{2} z}{r_{2}^{3}}
$$

Daraus folgt das Feld $\mathfrak{g}$ :

$$
\mathfrak{S}=-\operatorname{grad} V,
$$

oder ausführlich

$$
\begin{aligned}
& \mathfrak{S}_{x}=3 z\left(\mu_{1} \frac{x+D}{r_{1}^{5}}-\mu_{2} \frac{x-D}{r_{2}^{5}}\right), \\
& \mathfrak{S}_{y}=3 z y\left(\frac{\mu_{1}}{r_{1}^{5}}-\frac{\mu_{2}}{r_{2}^{5}}\right), \\
& \mathfrak{S}_{z}=3 z^{2}\left(\frac{\mu_{1}}{r_{1}^{5}}-\frac{\mu_{2}}{r_{2}^{5}}\right)+\frac{\mu_{2}}{r_{2}^{3}}-\frac{\mu_{1}}{r_{1}^{33}} .
\end{aligned}
$$

Zur Vereinfachung werde nun noch angenommen, daß der Dipol A (Abb.1) relativ zur Sonnenoberfläche ruhe, dann entsteht für einen Beobachter auf der Sonne durch die Bewegung von B allein ein induziertes elektrisches Feld vom Betrage

$$
\mathfrak{E}=\frac{1}{c}\left[\mathfrak{v}, \mathfrak{S}_{\mathrm{B}}\right]
$$

wobei $\mathfrak{K}_{\mathrm{B}}$ das Magnetfeld darstellt, welches sich für $\mu_{1}=0$ aus (2a) ergibt ${ }^{11}$.

Für ein Teilchen der Ladung $Z e$ gilt daher die Bewegungsgleichung ( $p$ relativistischer Impuls):

$$
\frac{d p}{d t}=Z_{e}\left(\mathfrak{s}+\frac{Z e}{c}[\mathfrak{v} \mathfrak{S}]\right. \text {. }
$$

Es ist im folgenden nicht unsere Absicht, alle möglichen Lösungen von (4) aufzusuchen und zu diskutieren. Wir wollen vielmehr zeigen, daß es überhaupt Lösungen gibt, die zu Teilchenbeschleunigungen im Bereich der Höhenstrahlungsenergien führen und die zugleich den Bewegungsvorgang voll zu übersehen gestatten. Dies scheint uns wichtig, da bisher kein Mechanismus bekannt war, der die Aussendung von Ultrastrahlungsteilchen durch die Sonne wirklich hat verstehen lassen.

Es soll darum hier unter allen möglichen Lösungen von (4) nur die einfachste und analytisch streng integrierbare angegeben und diskutiert werden. Natürlich ist es nicht unsere Meinung, daß dieses etwa der einzige in der Natur realisierte Vorgang dieser Art ist. Wir werden im zweiten Teil dieser Arbeit die Beschleunigungsvorgänge unter allgemeineren Gesichtspunkten diskutieren und zeigen, daß diese auch in komplizierteren Fällen möglich sind und wahrscheinlich gerade dann auftreten werden. Hier soll nur die prinzipielle Möglichkieit der Partikelbeschleunigung zu hohen Energien an einem besonders durchsichtigen Beispiel, das in vollem Umfang analytisch streng $\mathrm{zu}$ behandeln ist, vorgeführt werden.

Die Integration der Bewegungs gleichung im einfachsten Falle

Aus den Gln. (2a) entnimmt man, daß offenbar besonders einfache Verhältnisse vorliegen, wenn es Teilchenbahnen gibt, die nur in der Tangentialebene zur Sonnenoberfläche $(z=0)$ verlaufen. Dann nämlich verschwinden die Feldkomponenten $\mathfrak{S}_{x}$ und $\mathfrak{S}_{y}$ für beliebige Werte von $\mu_{1}$ und $\mu_{2}$. Außerdem aber sieht man, daß $\mathfrak{S}$ sogar zu Null werden kann, wenn $\mu_{1}=\mu_{2}$ und $r_{1}=r_{2}$ ist. Letzteres ist allerdings nur längs der $y$-Achse, also auf dem Mittellot zur Verbindungslinie der Fleckzentren, möglich.

11 Man kann ebensogut annehmen, daß beide Flekken sich mit gleicher Geschwindigkeit aufeinander zubewegen. 
Da andererseits in der $x$ - $y$-Ebene überall das Magnetfeld nur eine einzige nicht verschwindende Komponente in Richtung von $z$ besitzt, der Geschwindigkeitsvektor hingegen in Richtung der negativen $x$-Achse weist, so ist das elektrische Feld $E$ nach (3) in Richtung von $y$ orientiert.

Es ist damit unmittelbar klar, daß ein Teilchen, das sich einmal auf der $y$-Achse in Richtung dieser Geraden bewegt, diese nicht mehr verläßt, da es einerseits durch das Magnetfeld nicht mehr beeinflußt wird $(H=0)$, und andererseits die an ihm angreifende Kraft $Z e E$ es genau in dieser Richtung beschleunigt.

Wir können deshalb durch Spezialisierung der Differentialgleichung (4) für die Bewegung in der $\dot{y}$-Richtung die Gleichung ableiten:

$$
\begin{gathered}
\left(x=z=0 ; \dot{x}=\dot{z}=0 ; \ddot{x}=\ddot{z}=0 ; \mu_{1}=\mu_{2}=\mu\right) \\
m_{0} \frac{d}{d t} \frac{\dot{y}}{\sqrt{1-\dot{y}^{2} / c^{2}}}=\frac{Z e v \mu}{c} \frac{1}{\sqrt{y^{2}+D^{23}}} .
\end{gathered}
$$

Hierbei ist $m_{0}$ die Ruhemasse des zu beschleunigenden Teilchens.

Die Integration der Gl. (5) gelingt in bekannter Weise durch Multiplikation mit $\dot{y}$ auf beiden Seiten:

$$
\frac{m_{0} c^{2}}{\sqrt{1-\dot{y}^{2} / c^{2}}}=\frac{Z e v \mu}{c D^{2}}\left(\frac{y_{l}^{\prime} D}{\sqrt{1+y^{2} / D^{2}}}+E\right) .
$$

Wenn wir annehmen, daß das Teilchen in der Entfernung $y_{0}$ vom Zentrum startet, so besitzt es demnach beim Verlassen der Sonne die Energie:

$$
E=\frac{Z e v \mu}{c D^{2}}\left(1-\frac{y_{0} / D}{\sqrt{1+y_{0}^{2} / D^{2}}}\right) \text {. }
$$

Die weitere Integration der Bewegungsgleichung (5) führt auf elliptische Integrale.

Aus (5) folgt nämlich

$$
\dot{y} / c=\frac{\sqrt{\left(1+\frac{a y / D}{\sqrt{1+y^{2} / D^{2}}}-\frac{a y_{0} / D}{\sqrt{1+y_{0}^{2} / D^{2}}}\right)^{2}}-1}{1+\frac{a y_{l}^{\prime} D}{\sqrt{1+y^{2} / D^{2}}}-\frac{a y_{0} / D}{\sqrt{1+y_{0}^{2} / D^{2}}}},
$$

mit der Abkürzung:

$$
a=\frac{Z e v \mu}{m_{0} c^{3} D^{2}} .
$$

Der zeitliche Ablauf der Bewegung des Teilchens kann damit dem Ausdruck entnommen werden

$$
\begin{aligned}
t= & \frac{D}{c a} \int_{y_{0}}^{y} d\left(\frac{a y}{D}\right) \\
& \frac{1+\frac{a y / D}{\sqrt{1+y^{2} / D^{2}}}-\frac{a y_{0} / D}{\sqrt{1+y_{0}^{2} / D^{2}}}}{\sqrt{\left(1+\frac{a y / D}{\sqrt{1+y^{2} / D^{2}}}-\frac{a y_{0} / D}{\sqrt{1+y_{0}^{2} / D^{2}}}\right)^{2}-1}} .
\end{aligned}
$$

Daraus ergibt sich die für den Bewegungsablauf charakteristische Zeit $t_{0}$ :

$$
t_{0}=\frac{D}{c a}=\frac{m_{0} c^{2} D^{3}}{Z e v \mu} .
$$

Die Größe $t_{0}$ stellt bis auf Faktoren der Größenordnung Eins diejenige Zeit dar, die das Teilchen etwa benötigt, um den für den Beschleunigungsvorgang wesentlichen Teil des Fleckfeldes zu durcheilen.

Diskussion der Ergebnisse für das bipolare Dipolfeld

Die im letzten Abschnitte eingeführten Annahmen über die Feldverläufe sind bei den auf der Sonne auftretenden Magnetfeldern natürlich nie in mathematischer Strenge erfüllt, insbesondere ist der von uns diskutierte Spezialfall $\mu_{1}=\mu_{2}=\mu$ nur ganz selten realisiert. Weiter ist $\mathrm{zu}$ bedenken, daß es nur in einer gewissen Näherung erlaubt ist, die Magnetfelder der Flecken durch solche von Dipolen zu idealisieren. Nach Untersuchungen von $\mathrm{Ch}$ a p man ${ }^{9}$ ist man mindestens ebenso berechtigt, das Feld eines Sonnenflecks durch das eines magnetischen „Einzelpols“ approximativ darzustellen.

Schließlich aber ist zu beachten, daß die realen Flecken natürlich keine Singularitäten in den Fleckzentren besitzen, sondern daß diese vielmehr im Mittelbereich über weite Gebiete hinweg (einige $1000 \mathrm{~km}$ ) einen Maximalwert der Feldstärke von einigen Tausend Gauß haben können.

Bei anderen Annahmen über die Feldverläufe lassen sich leider die Bewegungsgleichungen der Teilchen auch in ausgesuchten Spezialfällen nicht so einfach integrieren wie in dem eben besprochenen Falle. Man muß dann andere Betrachtungen durchführen, die weiter unten besprochen werden. Wir wollen hier nur feststellen, daß allein aus dimensionsanalytischen Gründen für einen Modellvorgang, der dem von Abb. 1 nachgebildet ist, bei dem aber die Dipole A und B durch zwei gleich- 
namige Einzelpole ersetzt sind, die Energie, die ein Teilchen bei Beschleunigungsvorgängen in diesem Felde gewinnen kann (hinsichtlich des Mechanismus vergleiche hierzu den folgenden Abschnitt), den Wert besitzen muß:

$$
E \cong \frac{Z e v p}{c} \frac{p}{D}
$$

Hierbei sei $p$ die „Polstärke“ der Flecken. Wir werden sogleich zeigen, daß sich in diesem Falle etwa die gleichen Teilchenenergien ergeben wie nach Gl. (7).

Zunächst mögen die nach (7) zu erwartenden Energiewerte abgeschätzt werden.

Bei Wanderungsgeschwindigkeiten von etwa einem Fleckdurchmesser pro Tag, kann man mit Werten von $v$ rechnen

$$
v / c \sim 10^{-6} .
$$

Für die ,magnetischen Momente“ von Flecken" setzt man an:

$$
\mu \approx \frac{4 \pi}{3} \bar{H} R^{3} \sim 10^{31} \quad \mathrm{Gau} \beta \mathrm{cm}^{3}
$$

( $H=$ magnet. Feldstärke; $R=$ Fleckradius $10^{9} \mathrm{~cm}$ ),

$$
\bar{H} \approx 2000 \mathrm{Gau} \beta .
$$

Als Abstand wählen wir $D=1,5 \cdot 10^{9} \mathrm{~cm}$. Mit diesen Zahlwerten ergibt sich für $E$ nach (7):

$$
E \approx 10^{9} \mathrm{eV} \text { (für } y_{0} / D=0,3 \text { und } Z=1 \text { ). }
$$

Diese Zahlwerte sind natürlich nur als grobe Richtwerte zu betrachten, die nach beiden Seiten beträchtlich abweichen können. So sind, wie schon oben erwähnt, Fleckfelder mit einem äquivalenten $\mu=10^{31} \mathrm{Gau} ß \mathrm{~cm}^{3}$ keine Seltenheit; auch kommt es ziemlich häufig vor, daß zwei verschiedenpolige Flecken, die erst getrennte Bereiche vorstellten, sich nahe kommen und äußerlich zu einem einzigen Fleck verschmelzen, so daß für das Auge die bipolare Natur des Flecks gar nicht mehr erkennbar ist.

Die $D$-Werte können dann um Faktoren von 2-4 kleiner sein als die oben angenommenen Werte. Schließlich aber stellt die Wanderungsgeschwindigkeit $v / c=10^{-6}$ einen Wert dar, der sich aus Mittelbildungen über aktive Flecken bei Beobachtungen von Tag zu Tag ergeben. Es ist ziemlich wahrscheinlich, daß kurzfristig höhere $v / c$-Werte auftreten.
Jedenfalls aber zeigt die Abschätzung (16), daß unter den hier diskutierten Umständen Teilchen der Ladung $Z e$ Energien vom Betrage $Z \cdot 10^{9} \mathrm{eV}$ erhalten können.

Die oben angeführten Argumente machen es weiterhin plausibel, daß diese Energiewerte in besonderen Fällen leicht um eine Zehnerpotenz höher liegen können, und es scheint uns damit verständlich, daß die von $\mathrm{F}$ orbush, Ehmert u. a. beobachteten Effekte wirklich von Teilchen ausgelöst wurden, die ihre Energien von den veränderlichen Magnetfeldern der Sonnenflecken bezogen.

Daß diese Energieabschätzung dabei weitgehend von der speziellen Annahme über den Feldverlauf im Fleck selbst unabhängig ist, zeigt ein Vergleich der Gln. (7) und (12), wenn man dabei vom „Geometriefaktor"

$$
1-\frac{y_{0} / D}{\sqrt{1+y_{0}^{2} / D^{2}}} \text { absieht. }
$$

Da sich ja $p$ näherungsweise ergibt aus der Beziehung: $p \sim \pi R^{2} H$, so sieht man, daß sich (12) aus (7) ableiten läßt, indem man den letzteren Ausdruck mit dem Faktor $3 D / 4 R$ multipliziert. Dieser aber ist unter Berücksichtigung von (14) und (15) von der Größenordnung Eins, so daß wir nach (7) zu den gleichen $E$-Werten gelangen.

Weiterhin sei vermerkt, daß sich mit den hier angenommenen Zahlwerten nach (11) für die „Beschleunigungszeit" $t_{0}$ ergibt:

$t_{0}=\frac{m_{0} c^{2}}{Z e} \frac{D^{3}}{v \mu} \sim \frac{1}{30} \sec \quad($ für Protonen $Z=1)$.

Diese Zeit ist sehr klein gegen die Zeiträume, in denen sich die Feldverläufe von $H$ und $E$ merklich ändern, so daß jedenfalls damit unsere Betrachtungen (1) bis (11) nachträglich gerechtfertigt sind, denen die stillschweigende Annahme zugrunde liegt, daß während des Beschleunigungsvorganges die elektrischen und magnetischen Felder als konstant angesehen werden können.

Schließlich werde kurz noch der Einfluß der Bremsung durch die Sonnenmaterie diskutiert. Es ist klar, daß es für diesen Effekt wesentlich auf den Verlauf der Gesamtelektronendichte $N_{\mathrm{e}}^{*}$ (gebundene + freie Elektronen) am Sonnenrand ankommt. Wir haben deshalb in Abb. 2 diese dargestellt und die daraus folgende Bremsung von Protonen im „Minimum der Ionisation“ angegeben. 
Diesen geringen Bremsverlust $(\sim 3000 \mathrm{eV} / \mathrm{cm}$ in Luft unter Normalbedingungen) erleiden Protonen zwar erst, wenn sie schon Energien der Größenordnung $5 \cdot 10^{8} \mathrm{eV}$ besitzen. Für energieärmere wächst er stark an und erreicht etwa den 200fachen Wert für Protonen einer Energie von $\sim 1 \mathrm{MeV}$; um etwa einen weiteren Faktor 100 vergrößert er sich für ganz langsame Protonen der Energie 100-1000 eV.

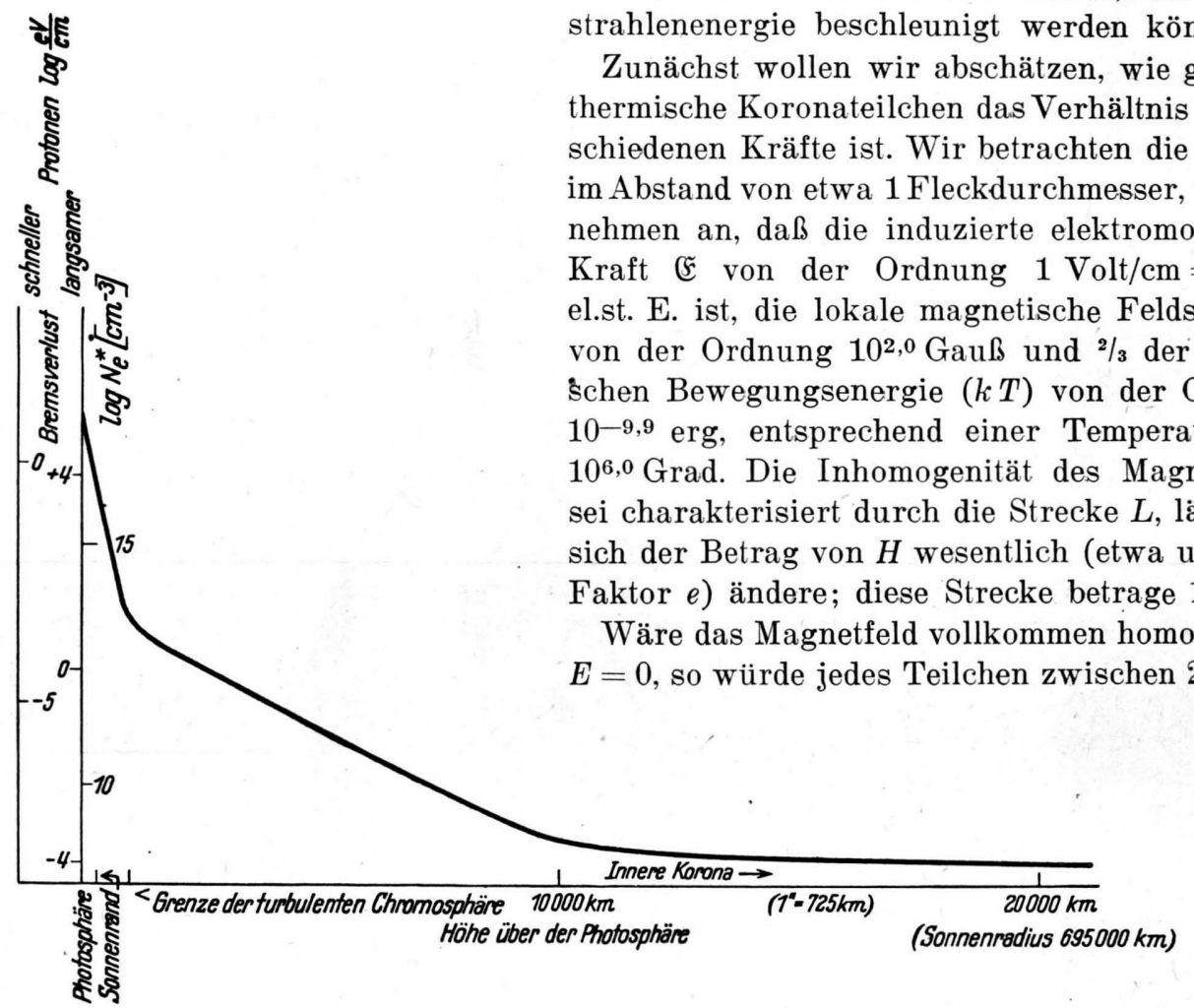

Abb. 2. Gesamtelektronendichte und Bremsverlust von Protonen in Abhängigkeit von der Höhe über der Photosphäre.
Die Berücksichtigung dieser Verhältnisse zeigt im Zusammenhang mit Abb.2, daß bei elektrischen Feldstärken der Größenordnung $1 \mathrm{eV} / \mathrm{cm}$, wie sie in sehr aktiven Fleckfeldern auftreten, Beschleunigungen vom Bereiche der mittleren Chromosphäre ab, d.h. 3000-5000 km oberhalb der Photosphäre, stattfinden können. Jedenfalls überwiegt von da ab selbst bei langsamen Protonen die beschleunigende elektrische Kraft den Einfluß der Bremsung durch die Sonnenmaterie.

Für höher geladene Teilchen (Fe XVI oder NiXIV) setzen entsprechend günstige Verhältnisse bei etwas größeren Höhen, sicher aber von Bereiche also, in dem diese Teilchen ohnehin erst in merklicher Anzahl auftreten.

Die Beschleunigung längs ins Unendliche führender magnetischer $\mathrm{Kraftlinien}$

In diesem Abschnitt soll gezeigt werden, wie geladene Teilchen längs der magnetischen Feldlinien, die ins Unendliche laufen, auf Ultrastrahlenenergie beschleunigt werden können.

Zunächst wollen wir abschätzen, wie groß für thermische Koronateilchen das Verhältnis der verschiedenen Kräfte ist. Wir betrachten die Korona im Abstand von etwa 1 Fleckdurchmesser, und wir nehmen an, daß die induzierte elektromotorische Kraft 15 von der von der Ordnung $10^{2,0} \mathrm{Gau} ß$ und $2 / 3$ der thermischen Bewegungsenergie $(k T)$ von der Ordnung 10-9,9 erg, entsprechend einer Temperatur von $10^{6,0}$ Grad. Die Inhomogenität des Magnetfeldes sei charakterisiert durch die Strecke $L$, längs der sich der Betrag von $H$ wesentlich (etwa um einen Faktor $e$ ) ändere; diese Strecke betrage $10^{9,5} \mathrm{~cm}$.

Wäre das Magnetfeld vollkommen homogen und $E=0$, so würde jedes Teilchen zwischen 2 Stößen der unteren Grenze der Korona ab ein, in einem

seinen Larmor-Kreis um eine feste magnetische Feldlinie beschreiben. Der Radius $R_{\mathrm{L}}$ desselben beträgt $m v c / Z e H=c \sqrt{2 m k T} / Z e H$. Für Protonen ergeben sich z. B. $4 \mathrm{~cm}$, für Elektronen $1 \mathrm{~mm}$, für 14-fach geladene Fe-Ionen $2 \mathrm{~cm}$. Wenn die Teilchen (Protonen oder Elektronen) sich dagegen senkrecht zum Magnetfeld mit Höhenstrahlenenergie bewegen, ist der Larmor-Radius für beide etwa $10^{4} \mathrm{~cm}$.

Wirkt nun außerdem ein elektrisches Feld dessen Betrag $\ll H$ ist, so wirkt die Komponente von $\mathfrak{E}$, die $\| \mathfrak{H}$ ist (wir nennen sie $E \|$ ), direkt beschleunigend. Das Teilchen gewinnt daher pro cm 
Weg längs der magnetischen Feldlinie $Z \mathrm{eV}$ Energie, falls $E \| 1$ Volt/cm beträgt. Die Komponente von $\mathfrak{F}$ dagegen, welche $\perp \mathfrak{H}$ ist (wir bezeichnen sie durch $E_{\perp}$ ), bewirkt nur eine Drift $\perp$ zu $\mathfrak{S}$ und $\perp$ ほ, mit der Geschwindigkeit $c E_{\perp} / H=10^{6} \mathrm{~cm} /$ $\mathrm{sec}=10 \mathrm{~km} / \mathrm{sec}$. In einem Koordinatensystem nämlich, das mit der Geschwindigkeit $\mathfrak{v}$ sich gegen die Sonne bewegt, ergibt die Lorentz-Transformation eine elektromotorische Kraft $\left[\frac{\mathfrak{v}}{c} \mathfrak{G}\right]$, so daß in dem $\perp \mathfrak{S}, \mathfrak{E}$ mit der Geschwindigkeit $c E_{\perp} / H$ driftenden Koordinatensystem $\mathfrak{\&}$ durch $\left[\frac{\mathfrak{v}}{c} \mathfrak{S}\right]$ gerade kompensiert wird. Im driftenden Koordinatensystem beschreibt das Teilchen (Ion oder Elektron) daher seine ungestörte Kreisbahn.

Die Beschleunigung beträgt für $E \|=1 \mathrm{Volt} / \mathrm{cm}$ für ein Proton $10^{11,8} \mathrm{~cm} / \mathrm{sec}^{2}$, für ein Elektron $10^{15,2} \mathrm{~cm} / \mathrm{sec}^{2}$, für ein $\mathrm{Fe}-\mathrm{XV}$-Ion $10^{11,4} \mathrm{~cm} / \mathrm{sec}^{2}$. Die Endgeschwindigkeit wird gemäß Gl. (17) erreicht in Zeiten einer Größenordnung von $10^{-1} \mathrm{sec}$ oder weniger. Die seitliche Drift spielt keine Rolle für die Bahn des Teilchens während des Beschleunigungsvorganges.

Die Wirkung der Inhomogenität des Magnetfeldes läßt sich im Falle $R_{\mathrm{L}} \nabla \ln |\mathfrak{S}| \ll 1$ auf folgende Art überblicken ${ }^{12}$. Das um eine magnetische Kraftlinie umlaufende Ion oder Elektron stellt (unabhängig vom Betrag oder Vorzeichen von $Z$ ) ein magnetisches Doppelblatt dar vom mittleren Moment

$$
\begin{aligned}
\mathfrak{M}=-\mathfrak{S} \cdot(\text { kinetische Energie } \perp \mathfrak{W}) \div H^{2} \\
=-\frac{|\mathfrak{M}|}{H} \mathfrak{S} .
\end{aligned}
$$

Dieses Moment bleibt auch bei der Bewegung in einem inhomogenen oder sich ändernden Magnetfeld konstant, d.h. der Larmor-Kreis umschließt eine gleichbleibende Anzahl magnetischer Feldlinien ${ }^{13}$. Die Kraft, die das Magnetfeld $H$ auf ein Moment $\mathfrak{M}$ ausübt, ist gegeben durch den Ausdruck

$$
\left(\mathfrak{M} \text { grad) } \mathfrak{S}=-\frac{|\mathfrak{M}|}{H}(\mathfrak{Q} \text { grad }) \mathfrak{S},\right.
$$

12 A 1 f yén, Ark. Mat., Astronom. Fysik, Ser. A 27, Nr. 22 [1940].

13 Die Fläche des' Larmor-Kreises ist unter diesen Umständen $\sim 1 / H$.

${ }_{14} \mathrm{Zu}$ denen die Kleinheit der Zentrifugalbeschleunigung infolge der Krümmung der magnetischen Feldlinien hinzutritt; die Winkelgeschwindigkeit längs $\mathfrak{S}$ muß klein sein gegen die um $H$. mit der $x$-Komponente

$$
\frac{|\mathfrak{M}|}{H}\left(H_{x} \frac{\partial H_{x}}{\partial x}+H_{y} \frac{\partial H_{x}}{\partial y}+H_{z} \frac{\partial H_{x}}{\partial z}\right) .
$$

Für rot $\mathfrak{S}=0$ beträgt die $\mathrm{Kraft} M \nabla H$, sie ist also von der Größenordnung $k T / L \approx 1^{-19,5} \mathrm{dyn}$, und für ein Ion und Elektron zusammen das Doppelte hiervon. Die Komponente dieser Kraft, welche senkrecht zu $H$ ist, bewirkt wie im Feld der elektrischen Feldstärke eine Drift, welche aber noch sehr viel langsamer ist, da $k T / L \ll e E$ ist. Die Komponente $\| \mathfrak{H}$ ist jedenfalls $\ll e E$, kann also gegen diese außer Betracht bleiben. Dagegen ist sie bei hoher Ionisation von der Größenordnung der Schwerkraft. Daher sollte diese Kraft ein gewisses Raumgebiet über aktiven Flecken weitgehend materiefrei halten können.

Es zeigt sich also, daß im Bereich der gemachten Approximationen ${ }^{14}$, d.h. jedenfalls in einer sehr weiten Umgebung der Flecken, geladene Teilchen nur längs der magnetischen Kraftlinien beschleunigt werden. Um die Sonne zu verlassen, müssen sie daher auf Feldlinien starten, welche in einer noch zu bestimmenden Annäherung ins Unendliche führen.

\section{Der Verlauf der magnetischen Kraftlinien über Flecken}

a) Stationäre Flecken

Die Beobachtung zeigt, daß das Flächenintegral $\int H_{Z} d F\left(H_{Z}\right.$ Normalkomponente der magnetischen Feldstärke) bei den Einzelflecken einer bipolaren Fleckengruppe im allgemeinen ganz verschiedene Werte annimmt. Mindestens die von dem größeren Fleck ausgehenden magnetischen Feldlinien müssen daher größtenteils in einer so weiten Umgebung des Flecks zur Oberfläche zurückkehren, daß die magnetische Feldstärke unter die Meßgrenze rückt ${ }^{15}$. Nehmen wir an, daß diese bei ${ }^{1 / 20}$ der mittleren Feldstärke eines großen Flecks liegt, so muß die Fläche der fraglichen Umgebung mindestens 20-mal so groß sein, d.h. von der Ordnung $10^{10} \mathrm{~km}^{2}$. Wenn andererseits die Feldlinien des schwächeren Flecks der Gruppe (deren magnetische Polarität der des Hauptflecks entgegengesetzt angenommen sei) größtenteils in den

15 Man darf vermutlich annehmen, daß die die Flekken umgebenden chromosphärischen Fackelgebiete, wie sie auf den Spektroheliogrammen im Lichte der Linie K erscheinen, die Ausdehnung der Magnetfelder im Niveau der Chromosphäre anzeigen. 
Hauptfleck hineinliefen, sollte man irgendeine Asymmetrie der Feldverteilung erwarten; diese ist nie beobachtet worden, doch mag dies an der Anlage der bisherigen Beobachtungen liegen.

Viele Beobachtungen an Protuberanzen in der Nähe von Flecken sprechen dafür, daß die Bewegungen einzelner Teilelemente derselben von magnetischen Feldlinien geführt werden ${ }^{16}$, die bogenförmig hoch über der Sonnenoberfläche verlaufen und in einen Fleck einmünden.

Endlich kann man leicht sehen, daß eine Abschirmung des Magnetfeldes einer stationären Fleckengruppe gegen die Korona hin durch entsprechende Flächenströme in der Chromosphäre auf unmöglich hohe magnetische Druckgradienten führen würde. Die Höhe der Chromosphäre (etwa $10000 \mathrm{~km}$ ) ist nämlich klein gegen die Ausdehnung großer Flecken und vergleichbar mit derjenigen kleiner Teilflecken einer Gruppe. Wegen $\operatorname{div} \mathfrak{S}=0$ müßte dann in der Chromosphäre $|\mathfrak{S}|$ überall von derselben Ordnung wie in der Photosphäre sein. Da $\dot{\hat{s}}$ sicher $\ll i$ ist, müßte $\mathfrak{t} / c=\operatorname{rot} \mathfrak{S} / 4 \pi$ sein, und der magnetische Druckgradient wäre

$\mathfrak{f}=\frac{1}{4 \pi}[\mathfrak{S} \operatorname{rot} \mathfrak{S}] \approx \frac{\mathfrak{S}^{2}}{4 \pi} \div 10000 \mathrm{~km} \approx 10^{-4}\left[\frac{\mathrm{dyn}}{\mathrm{cm}^{3}}\right]$.

Diesem müßßte das Gleichgewicht gehalten werden durch eine Masse pro Flächeneinheit der Ordnung $\mathfrak{S}^{2} / 4 \pi g$, welche also einen Druck der Ordnung $\mathfrak{S}^{2} / 4 \pi \approx 10^{5} \mathrm{dyn} / \mathrm{cm}^{2}$ erzeugen würde. Dies ist aber um einige Zehnerpotenzen höher als der nach aller sonstigen Erfahrung in der Chromosphäre herrschende Druck. Flössen andererseits derartige abschirmende Ströme in der Photosphäre, so müßten die magnetischen Feldlinien im wesentlichen horizontal verlaufen, was ebenfalls aller Erfahrung widerspricht. Eine generelle $\mathrm{Ab}$ schirmung der Fleckfelder gegen die Korona scheint demnach ausgeschlossen zu sein, und für stationäre Flecken kann das Magnetfeld im Raum über den Flecken nicht wesentlich verschieden sein von dem, welches durch die Flecken im Vakuum erzeugt würde.

Es ergibt sich nun das Problem, für aktive (zeitlich veränderliche) Fleckengruppen ein Bild über die entsprechenden Änderungen von $\mathfrak{b}$ im Raum über den Flecken zu gewinnen.

16 T. G. C ow ling, Proc. Roy. Soc. [London] 183, 453 [1945]. b) Aktive Flecken

Wir betrachten zunächst einige Grenzfälle. Die einfachste und schon mehrfach behandelte Näherung* besteht darin, die Atmosphärenschichten über dem Fleck als unbewegliche leitende Schicht einer festen elektrischen Leitfähigkeit $\sigma$ mit der magnetischen Permeabilität 1 und der Dielektrizitätskonstante 1 anzusehen. Für einen Überblick genügt folgende Betrachtung. Bezeichnet man das Vektorpotential der magnetischen Feldstärke durch $\mathfrak{A}$ und nimmt an, daß wegen der hohen Leitfähigkeit keine Raumladungen auftreten, so gilt

$\Delta \mathfrak{U}-\frac{1}{c^{2}} \ddot{\mathfrak{A}}=-\frac{4 \pi}{c} \mathfrak{i}=\frac{-4 \pi \sigma}{c} \mathfrak{s}=+\frac{4 \pi \sigma}{c^{2}} \dot{\mathfrak{A}}$.

Nur für räumlich konstantes $\sigma$ kann man auch die entsprechende Gleichung für $\mathfrak{S}$ hinschreiben. Da die betrachteten Zeiträume $\gg \sigma^{-1}$ sind, bleibt im wesentlichen die Diffusionsgleichung für jede Komponente von $\mathfrak{A}$ übrig ${ }^{16}$. Wird das Anwachsen von $\mathfrak{S}$ durch die Zeitkonstante $\omega\left(\mathrm{sec}^{-1}\right)$ charakterisiert, so ist die Entfernung $\Delta x$, welche die Anderung von $\mathfrak{T}$ erreicht, größenordnungsmäßig gegeben durch die Beziehung

$$
\Delta x^{2} \approx \frac{c^{2}}{4 \pi \sigma \omega}, \quad \Delta x^{2} \omega^{2} \approx c^{2} \frac{\omega}{4 \pi \sigma}\left[\frac{\mathrm{cm}}{\mathrm{sec}}\right]^{2} .
$$

Die Störung pflanzt sich also fort mit der Geschwindigkeit $c \sqrt{\omega / 4} \overline{\pi \sigma}$. Bei bestimmten einfachen Symmetrieverhältnissen lassen sich die Lösungen auch explizit hinschreiben.

Setzt man $\omega=10^{-5}$ und $\sigma=10^{+12}$ (etwa entsprechend der ungestörten Photosphäre), so folgt für die Geschwindigkeit $10^{1,5} \mathrm{~cm} / \mathrm{sec}$ und für $\Delta x$ $10^{6,5} \mathrm{~cm}$.

Daß die Ausbreitung von $\mathfrak{A}$ und $\mathfrak{S}$ nicht mit Lichtgeschwindigkeit erfolgt, wird bewirkt durch die Induktionsströme, welche jeder Änderung von $\mathfrak{H}$ entgegenwirken. Die Wechselwirkung dieser Induktionsströme mit dem Magnetfeld bewirkt wieder einen Druck quer zu den magnetischen Feldlinien, dessen Kraftdichte gegeben ist durch

$$
\mathfrak{f}=\left[\frac{\mathfrak{i}}{c} \mathfrak{S}\right]=-\frac{\sigma}{c^{2}}[\dot{\mathfrak{A}} \operatorname{rot} \mathfrak{A}] \approx \frac{\mathfrak{S}^{2}}{4 \pi \Delta x},
$$

* S. z. B. K i e p e n h e u e r, Ann. d'Astrophysique 8, 210 [1945]; 9, 42 [1946]. 
bis auf einen Faktor der Größenordnung $\pi$. Um festzustellen, ob unsere Annahme einer ruhenden Schicht gerechtfertigt war, haben wir $\mathfrak{f}$, den magnetischen Druckgradienten, zu vergleichen mit dem Gradienten des Gasdrucks $|\nabla P|$. Nun ist $\mathfrak{S}^{2} / 4 \pi$ über Flecken von der Ordnung $10^{5}$. Andererseits ist

$$
|\nabla P|=\frac{d \log P}{d r} P=\frac{P}{h},
$$

wobei $h$ (die sòg. homogene Dicke der Schichtung) in der Photosphäre und der unteren Chromosphäre etwa $100 \mathrm{~km}$, in der mittleren und oberen Chromosphäre etwa $1000 \mathrm{~km}$, in der Korona sogar größenordnungmäßig $100000 \mathrm{~km}$ beträgt. Der Gasdruck $P$ beträgt in derPhotosphäre $10^{5} \mathrm{dyn} / \mathrm{cm}^{2}$, in der unteren Chromosphäre etwa $10^{3}$, in der mittleren und oberen Chromosphäre $10^{2}$ bis $10^{-1}$.

Es folgt hieraus, daß die oft gemachte Annahme einer unbeweglichen leitenden Atmosphäre zwar unterhalb der Photosphäre berechtigt ist, oberhalb derselben aber nicht. Die eintretenden Bewegungen sind primär von der Art, daß die magnetische Flußänderung durch jeden materiellen Querschnitt klein bleibt; die durch die eintretendén Bewegungen induzierten elektromotorischen Kräfte wirken den durch die Änderung von $\mathfrak{S}$ induzierten entgegen, mit der Einschränkung allerdings, daß hierdurch nur die Komponenten von $\mathfrak{C} \perp \mathfrak{S}$ kompensiert werden können.

Es zeigt sich also, daß die Ausbreitung von Störungen des Magnetfeldes einer aktiven Fleckengruppe zwar im Photosphärenniveau und erst recht darunter sehr langsam erfolgt, in die Chromosphäre und erst recht in die Korona hinein aber weitaus rascher, als die Rechnung für eine ruhende Atmosphäre andeutet. Die Tatsache, daß nur Bewegungen und hierdurch induzierte elektromotorische Kräfte $\perp \mathfrak{L}$ primär hervorgerufen werden können, bedingt, daß die Gestalt des Féldes sich bei der Ausbreitung ändern muß. Es kommt hinzu, daß die eintretenden Bewegungen noch von der relativen Lage von $\mathfrak{H}$ und $\nabla P$ abhängen, Bewegungen $\perp \nabla P$ treten sehr viel leichter ein als solche $\| \nabla P$.

c) DiөAusbreitung der Feldstörungen ins Koronaniveau

Nun ist noch ein weiterer wesentlicher Umstand zu berücksichtigen. Die elektrische Leitfähigkeit im Magnetfeld hängt bei den Dichten, die in der Sonnenatmosphäre vorkommen, sehr stark ab von dem Winkel zwischen $\mathfrak{E}$ und $\mathfrak{h}$. Die elektrische Leitfähigkeit $\| \mathfrak{S}$ ist praktisch die gleiche wie die für $\mathfrak{S}=0$, während die Leitfähigkeit quer zum Magnetfeld dadurch reduziert ist, daß jedes Elektron und Ion zwischen zwei Stößen seinen Larmor-Kreis beschreibt und daher überhaupt nur durch Stöße quer zu $\mathfrak{S}$ fortschreiten kann. Das Maß dieser Behinderung hängt ab von der Zahl der Larmor-Umläufe zwischen zwei Stößen, und diese wieder vom Verhältnis $H / p_{\mathrm{e}}\left(p_{\mathrm{e}}\right.$ Elektronendruck); die Grenze liegt etwa bei $H / p_{\mathrm{e}} \approx 2$ Gauß $\mathrm{cm}^{2} / \mathrm{dyn}$. Die Verminderung von $\sigma$ für $\mathfrak{E}_{\perp} \mathfrak{S}$ beträgt schon in der Chromosphäre eine ganze Anzahl von Zehnerpotenzen.

Betrachtet man nun, wie das aus Gründen der Einfachheit gewöhnlich geschehen ist, zylindersymmetrische Magnetfelder, so ist $\mathfrak{E}$ stets $\perp \mathfrak{S}$, und es kommt nur die maximal reduzierte elektrische Leitfähigkeit in Betracht. Dies bewirkt, daß in solchen Fällen die induzierten Ströme schwach sind und die Ausbreitungsgeschwindigkeit des Feldes besonders groß ist.

Geht man auf die Diffusionsgleichung von $\mathfrak{A}$ zurück, so kann man sagen, daß die Komponenten von $\mathfrak{A}$, welche $\perp \mathfrak{S}$ sind, rasch diffundieren, die Komponente von $\mathfrak{A}$, die $\| \mathfrak{S}$ ist, nur langsam. In Fleckengruppen ohne besondere Symmetrieeigenschaften werden daher die Induktionsströme immer nur partiell das Fortschreiten eines Feldes $\mathrm{zu}$ hindern vermögen.

Als einfaches Modell denke man sich ein Bündel an den Enden verbundener, zur $x$-Aehse paralleler isolierter Metalldrähte, welche die Leitfähigkeit $\| x$ groß machen, während die Leitfähigkeit $\perp x$ sehr klein ist. Schicken wir nun durch eine flache Spule in der $x$ - $y$-Ebene, deren Durchmesser klein sei gegen die Ausdehnung des Bündels, einen Strom, so fließen induzierte Ströme nur in der $x$-Richtung. Demnach wird die Ausbreitung der $H_{x}$-Komponente des durch die Spule erzeugten Magnetfeldes überhaupt nicht affiziert, während die $H_{y}$-Komponente sich nur sehr langsam durch das Drahtbündel hindurch ausbreiten wird. Der Teil der $H_{z}$-Komponente, der von dem Vektorpotential $A_{y}$ herrührt, breitet sich mit Lichtgeschwindigkeit aus, während $\operatorname{der}$ von $A_{x}$ herrührende Teil von $H_{z}$ sich wieder nur sehr langsam ausbreitet $\left(A_{z}=0\right)$. Aus Symmetrie- 
gründen sind beide Anteile in der Spulenachse im Endzustand $(t=\infty)$ gleich.

Wäre die Achse der Spule $\| x$, so würde die Ausbreitung des Magnetfeldes nicht behindert, da keine Induktionsströme fließen würden. In diesem Fall ist $A_{x}=0$.

Das besprochene Modell bildet den Fall nach, daß das sich ändernde Störmagnetfeld sehr schwach ist gegen das schon vorhandene Magnetfeld, dessen Feldlinien die bevorzugten Strombahnen festlegen. Auf der Sonne sind in aktiven Fleckengruppen beide vergleichbar.

Endlich wird die gewöhnliche Theorie der elektrischen Leitfähigkeit unzuständig, wenn der zwischen 2 Stößen aus dem Feld gewonnene Impuls vergleichbar wird mit $\sqrt{3 m k T}\left(\approx 10^{-19}\right.$ für die Chromosphäre). Dies ergibt eine Grenze für $E$, die recht niedrig liegt. Berücksichtigt man nur die Stöße mit anderen Elektronen oder Ionen (Chromosphäre) und setzt den mittleren Stoßquerschnitt für thermische Elektronen $\mathrm{zu} \quad 1^{-13} \mathrm{~cm}^{2}$ an, so wird die freie Weglänge $10^{+13} / N_{\mathrm{e}} \mathrm{cm}$ und die Stoßzeit $\approx 10^{+5} / N_{\mathrm{e}}$ sec. Der Impulsgewinn zwischen zwei Stößen wird somit $\left(10^{-4} / N_{\mathrm{e}}\right) \cdot E$, wenn $E$ in el.st. Einheiten gemessen wird. In der mittleren Chromosphäre $\left(N_{\mathrm{e}}=10^{10}\right)$ liegt die Grenze somit bei $\dot{E}=10^{-5}$ oder 1 Volt $/ 300 \mathrm{~cm}$. Wird diese Grenze überschritten, so steigt die Leitfähigkeit abrupt an. Es liegt nahe, diesen Vorgang für das Auftreten chromosphärischer Eruptionen in aktiven Fleckengruppen verantwortlich zu machen ${ }^{17}$.

Man kann demnach damit rechnen, daß über aktiven Flecken vorzugsweise längs der magnetischen Kraftlinien elektrische Entladungen vorkommen, die ihrerseits wieder sekundäre Magnetfelder hervorrufen, welche quer zu dem primären Hauptfeld liegen.

In der Korona, welche nicht mehr als leitendes Kontinuum behandelt werden kann, ist das Mißverhältnis zwischen der Dichte der kinetischen und der potentiellen Energie der dort vorhandenen Materie gegen die magnetische Energiedichte über Flecken besonders groß. In der unteren Korona beträgt die Massendichte etwa $10^{-16} \mathrm{~g} / \mathrm{cm}^{3}$ (entsprech. einer Elektronendichte von $10^{8} \mathrm{~cm}^{-3}$ ), und die kinetische und die potentielle Energie betragen etwa $10^{15} \mathrm{erg} / \mathrm{g}$. Die hieraus resultierende Energiedichte von $10^{-1} \mathrm{erg} / \mathrm{cm}^{3}$ ist vergleichbar

${ }_{17}$ Vgl. hierzu Giovanelli, Monthly Notices Roy. astronom. Soc. 108, 163 [1948]. der magnetischen Energiedichte bei 1 Gauß magnet. Feldstärke. Über Flecken überwiegt die letztere also weitaus. Nun bedingt aber eine Behinderung der Ausbreitung eines Magnetfeldes durch Materie in jedem Fall eine entsprechende Rückwirkung auf die letztere (wie wir sie für die als Kontinuum anzusehenden tieferen Schichten schon diskutierten), und diese Rückwirkung wird immer von der Art sein, daß die Behinderung der Ausbreitung des Magnetfeldes reduziert wird. Wir haben gesehen, daß die magnetische Energie groß genug ist, um die gesamte über den Flecken vorhandene Materie wegzuschaffen; man darf also wohl annehmen, daß die Korona über Flecken die Ausbreitung variabler Magnetfelder nicht wesentlich behindert. Dies gilt mit der Einschränkung, daß die Massendichte gewisse Grenzen unterschreiten muß, die im folgenden Abschnitt begründet werden.

Wir schließen aus dieser Diskussion, daß die Magnetfelder über aktiven(komplizierteren) Flekkengruppen in jedem Moment auch oberhalb der Chromosphäre hinsichtlich der Unregelmäßigkeit ihrer Struktur, ihrer Intensität und ihrer zeitlichen Variabilität denen vergleichbar sind, die sich in einem vollkommenen Vakuum über der Photosphäre einstellen würden.* Das heißt nicht, daß das wirkliche Feld in seiner Gestalt dem Vakuumfeld ähnlich ist, sondern nur hinsichtlich seiner hervorgehobenen Eigenschaften; es ist sehr unwahrscheinlich, daß das Feld im Koronaniveau von den besprochenen verwickelten Vorgängen im Raum zwischen der Photosphäre und der Korona nicht beträchtlich verändert wird. Es besagt nur, daß oberhalb der Chromosphäre keine Lagebeziehung zwischen den Vektorfeldern $\mathfrak{A}$ und $\mathfrak{\mathfrak { A }}$ vorhanden sein soll, die ohne Berücksichtigung des Einflusses der Atmosphäre im Koronaniveau nicht bestehen würde. Wir werden demgemä $\beta$ das Vakuumfeld für die folgende Diskussion zugrundelegen und haben nur nachträglich zu verifizieren, daß die durch induzierte Ströme erzeugten Rückwirkungen nicht mit den Beobachtungen unverträglich sind.

* Anm. bei der Korrektur: Einen Beweis hierfür hat inzwischen A. Schl üte r gefunden im Zusammenhang mit theoretischen Untersuchungen über das elektrische und magnetische Verhalten von Elektronenplasmen, welche die in diesem Abschnitt durchgeführten Überlegungen in mancher Hinsicht weiterführen. Hrn. Schlüter sind wir für mehrere Diskussionen und für die Erlaubnis, von seinen Ergebnissen noch vor ihrer Veröff. (Z. Naturforschg., im Druck) Gebrauch zu machen, zu großem Dank verpflichtet. 
Die induzierten elektromotorischen $\mathrm{Kr}$ äfte

Denkt man sich nun einen Einzelfleck approximiert durch einen Dipol, der sich etwas unter der Oberfläche befindet, so ist klar, daß alle Kraftlinien in die Oberfläche zurücklaufen, bis auf die eine, die seńkrecht über dem angenommenen Dipol startet. Steht neben diesem Dipol ein zweiter von entgegengesetzter Polarität, aber gleichem Moment, so werden die Kraftlinien der ersten (bei dem sich die Quelle von $H$ oben befinden möge) teilweise zum zweiten (Senke oben) hinübergebogen. Die Feldlinie, die vom ersten Dipol ins Unendliche verläuft, bildet nun in größerer Entfernung einen Winkel von etwa $45^{\circ}$ zur Achse der beiden Dipole. Es ergibt sich also, daß in komplizierten Fleckengruppen die ins Unendliche laufenden Feldlinien stets irgendwelche Winkel zwischen $0^{\circ}$ und $90^{\circ}$ gegen die Oberfläche bilden werden.

Über die Lage der induzierten elektrischen Kraftlinien läßt sich folgendes sagen: Falls ihre Ursache in der Verstärkung oder Schwächung eines vorhandenen Flecks besteht, umschließen die induzierten elektrischen Feldlinien die Achsen des Flecks und sind somit horizontal. Falls ein magnetischer Dipol sich mit der Geschwindigkeit $v$ bewegt, so ist $E$ senkrecht zu $v$ und zu der von diesem Dipol allein erzeugten magnetischen Feldstärke. In komplizierteren aktiven Fleckengruppen sind also stets Komponenten von E in Richtung der ins Unendliche führenden magnetischen Kraftlinien des magnetischen Gesamtfeldes zu erwarten. Zwei durchsichtige Spezialfälle sind die folgenden: 1 . Seitlich von einem bipolaren Doppelfleck steht ein dritter, der entweder sich in der Richtung zum Doppelfleck bewegt oder sein Moment ändert. \& ist dann horizontal gerichtet, während die von jedem $\mathrm{Pol}$ des Doppelflecks ins Unendliche verlaufenden Feldlinien etwa einen Winkel $\pi / 4$ zur Oberfläche und zu 〔 bilden. 2. Der eine Pol eines bipolaren Doppelflecks bewegt sich senkrecht zur Verbindungslinie der beiden Pole. Auch hier hat $\mathfrak{\xi}$ eine wesentliche Komponente in Richtung der vom anderen Pol ins Unendliche verlaufenden Feldlinie ${ }^{18}$. .

18 Nach freundl. Mitt. von Hrn. Wald me i e r hat dieser in einem Vortrag auf der Zeeman-Konferenz in Amsterdam (1946) diese Verhältnisse im Hinblick auf den Energiehaushalt der Korona diskutiert. Vgl. Physica, Dezember 1946.
Es zeigt sich also, daß die geometrischen Bedingungen der relativen Lage von $\mathfrak{F}$ und $\mathfrak{S}$, welche für die Beschleunigung auf Ultrastrahlungsenergie notwendig erfüllt sein müssen, in komplizierten aktiven Fleckengruppen oft vorliegen werden.

Solange die freien Weglängen kleiner oder noch vergleichbar mit den betrachteten Dimensionen sind, wird die Rückwirkung der in der leitenden Materie induzierten Ströme Beschleunigungen einzelner Teilchen verhindern; sobald aber Massenbewegungen etwa sämtlicher Ionen einsetzen, begrenzt die zur Verfügung stehende magnetische Energie die Intensität. Wir wollen fragen, welche elektrische Stromdichte $i$ der entstehenden Ultrastrahlungsteilchen noch keine wesentliche Rückwirkung auf die solaren Magnetfelder gibt; diese Rückwirkung mißt ja die übertragene Energie. Dies gibt für die Stromstärke $J$

$$
J<\frac{c}{2} \bar{r} H \quad \text { (el. st. E.) }
$$

und für die Stromdichte $i$

$$
i<\frac{c}{2 \pi} \frac{\bar{r}}{a} \frac{H}{a} .
$$

Dabei ist $\bar{r}$ der Abstand der Strombahn von der Sonnenoberfläche und $a$ der Radius der Strombahn.

Wir fragen jetzt, von einem wie großen Teil des Flecks die Teilchen ins Unendliche entweichen. Hierzu betrachten wir zunächst die Fläche, auf welchẻr der Larmor-Radius gleich dem Abstand vom Fleck wird, und überlegen, ein wie großer Teil der vom Fleck ausgehenden magnetischen Kraftlinien diese Fläche noch erreicht. Für Ionen wird der Radius $R_{\mathrm{L}}$ etwa $10^{1,5} / \sqrt{H} \mathrm{~cm}$ sein, und $H$ nimmt in größerer Entfernung $r$ ab etwa $(L / r)^{3}$, wo $L$ der Abstand der entgegengesetzten Pole der angenommenen Dipole ist. Dieser darf $\mathrm{zu}$ etwa $10^{10} \mathrm{~cm}$ angenommen werden und die Feldstärke im Abstand $L$ zu etwa $10^{2}$ Gauß ${ }^{19}$. Dies gibt für $r=10^{1} \mathrm{~L}, H \approx 10^{-1}$ und $R_{\mathrm{L}} \approx 10^{2}$, das Feld würde also die Ionen bis in riesige Entfernungen führen, wenn nicht die ganze Betrachtung nur für die mit der Sonne rotierende Korona sinnvoll wäre. Man muß also fragen, welcher Bruchteil der Feldlinien so weit reicht. Offenbar

19 Tatsächlich ist der Abfall mit der Entfernung zunächst langsamer, und andererseits die Feldstärke im Abstand $L$ wohl etwas kleiner. 
fällt die Zahl der Feldlinien, welche die Entfernung $r$ vom Dipol erreichen $\sim 1 / r$. Man darf also rechnen, daß im ganzen etwa $1 / 100$ bis $1 / 10$ der von einem Einzelfleck ausgehenden Feldlinien die Grenze der Korona erreichen, wenn in dieser, wie hier vorausgesetzt wird, keine abschirmenden Ströme fließen.

Die Größe $a^{2}$ in der oben stehenden Grenzbeziehung für $i$ wird demgemäß zu $10^{17} \mathrm{~cm}^{2}$ angesetzt werden dürfen. Mit $\bar{r}=10^{10} \mathrm{~cm}$ gibt dies für $i$ die obere Grenze $10^{5}$ el.st. E. oder etwa $10^{15}$ Protonen pro $\mathrm{cm}^{2}$ und sec von fast Lichtgeschwindigkeit. Wenn also über einem aktiven Fleck die Elektronendichte lokal den Wert $10^{5} \mathrm{~cm}^{-3}$ wesentlich unterschreitet, so kann dort $E \|$ groß genug zur Produktion von Ultrastrahlung werden.* Die Grenzintensität ist um Zehnerpotenzen höher, als der bei einem Höhenstrahlausbruch auf der Erde ankommenden Anzahl von Höhenstrahlteilchen entspricht.. Offenbar ist die in Form von Ultrastrahlung emittierte Energie ja auch ein winziger Bruchteil der verfügbaren magnetischen Energie.

Die Elektronen sind gegenüber den Ionen benachteiligt, insofern sie sich weniger leicht von den Feldlinien loslösen können. Dies macht verständlich, warum von der Sonne vorzugsweise positive Ionen zu uns kommen. Umgekehrt sind die Metallionen (FeXV usw.) gegenüber den Protonen noch etwas bevorzugt.

Nach diesen Überlegungen wäre zu erwarten, daß oft solche Bedingungen herrschen, daß nur hochgeladene Metallionen die Energie erreichen, die sie als Ultrastrahlteilchen zur Erdoberfläche gelangen läßt. Dann sollte es eine um Größenordnungen höhere Zahl von Teilchen mit Energien der Ordnung $10^{8}$ Volt und weniger geben. Die Energiebilanz würde dies ohne weiteres erlauben, wie man leicht nachrechnet. Nach freundl. Mitt. von Hrn. Ehmert liegen von seiten der Beobachtungen Anzeichen hierfür vor. Man sollte demnach in hohen magnetischen Breiten in größerer Höhe über dem Erdboden Primärteilchen dieser Energie von der Sonne erwarten.

Die Rückwirkung der in der Korona induzierten Wirbelströme auf die primären Vorgänge in

\footnotetext{
* Diese Grenze fällt ungefähr zusammen mit der, die sich aus der Forderung ergibt, daß die Störung des Magnetfelds sich (wie im Vakuum) praktisch mit Lichtgeschwindigkeit fortpflanzt (A. Schl üter, vgl. Anm. b. d. Korr.).
}

den Flecken und auf die Feldverteilung im Zwischengebiet tendiert dahin, die induzierten elektromotorischen Kräfte zu schwächen; aber die magnetischen Feldänderungen, die wir in der Photosphäre beobachten, enthalten diese Rückwirkung schon. Es scheint, daß normalerweise eine automatische Kompensation dieser Art das Entstehen von Ultrastrahlung verhindert, und daß nur kräftige Störungen es ermöglichen; tatsächlich entsteht ja auch nicht über jeder aktiven Gruppe Ultrastrahlung.

Diese Verhältnisse und die vorherige Abschätzung der Partikeldichten läßt auch die Bedeutung der zuvor hervorgehobenen Tatsache erkennen, daß im Magnetfeld der Flecken die Schwerkraft scheinbar kompensiert sein kann. In Bereichen mit normaler Koronadichte könnte der beschriebene Mechanismus nicht funktionieren, da viel zu hohe Stromdichten entstehen würden. Über den Flekken dagegen können, wie wir schon sahen, geeignete Störungen den Raum leerfegen, mindestens in der Umgebung der Feldlinien, die in große Entfernung vom Fleck führen. Hierdurch erklärt sich wohl die Beobachtungstatsache, daß die Höhenstrahlausbrüche gewöhnlich von Störungen (chromosphärische Eruptionen) und normaler Korpuskularstrahlung (Laufzeit etwa 1 Tag) eingeleitet werden.

Die Entstehung von Ultrastrahlung i m Kosmos

Es ist weiterhin die Frage zu diskutieren, ob auf anderen Sternen Ionen mit noch höherer Energie als auf der Sonne entstehen könnten. Dimensionsmäßig kann die Energie geschrieben werden in der Form

$$
Z e H \cdot \frac{v}{c} \cdot \text { Länge. }
$$

Die Größenordnung von $H$ ist auch auf den Sternen, auf denen bisher Magnetfelder beobachtet worden sind, nicht merklich anders als auf der Sonne. Dafür kann aber $v$ bzw. $\dot{H} / H$ bei rasch rotierenden Sternen leicht eine oder zwei Größenordnungen größer sein. Ebenso sind die Durchmesser der Sonnenflecken nur ein kleiner Bruchteil des Sonnendurchmessers. Bei den jetzt von $\mathrm{B}$ a b c o c k beobachteten intensiven Magnetfeldern auf rasch rotierenden Sternen ist die dem Fleckdurchmesser entsprechende Größe offenbar von der Ordnung des Sternradius. Ob es sich dabei um 
Felder nach der Art von Dipolfeldern handelt, muß als völlig offen bezeichnet werden; einmal scheint eines der von Babcock beobachteten Felder zeitlich nicht konstant zu sein, sondern seine Polarität zu wechseln, und zum anderen muß auch die Frage, ob die Sonne überhaupt ein allgemeines Magnetfeld von Dipolcharakter besitzt, noch als durchaus offen angesehen werden (es könnte z.B. auf die Photosphäre beschränkt sein). Es ist also plausibel, anzunehmen, daß es Sterne gibt, auf denen die erreichte Energie um mehrere Zehnerpotenzen über der auf der Sonne anzunehmenden liegt.

Es liegt nahe, die Frage aufzuwerfen, ob die gesamte Ultrastrahlung auf den Sternen entstehen könnte. Das Mißverhältnis der scheinbaren Helligkeiten sämtlicher Sterne und Sternsysteme zusammengenommen auf der einen Seite und der scheinbaren Helligkeit der Sonne auf der anderen Seite, scheint diesen Gedanken aber fast auszuschließen. Die Sonne dürfte höchstens $10^{-10}$ ihrer gesamten Energie in Form von Ultrastrahlung emittieren $^{20}$, während im interstellaren Raum die Energiedichte der Ultrastrahlung vergleichbar mit der des Lichts sein wird.

Man würde darum zunächst vermuten, daß dieses „Mißverhältnis“ bei günstigeren Objekten als der Sonne, wenn auch nicht in vollem Umfange, so doch wenigstens in seinem qualitativen Charakter erhalten bleibt.

Es gibt jedoch einen Ausweg, der die Behebung dieser Schwierigkeit zu gestatten scheint.

Während nämlich das Licht sehr weit entfernter Sterne durch die mit der Expansion des Weltalls verbundene Rotverschiebung der Spektrallinien schon bei „relativ kleinen“ Geschwindigkeiten aus dem sichtbaren Bereiche herausrückt und damit dessen Energie unter die Meßbarkeits-

20 A. Ehmert, Vortrag beim Kolloquium in Weißenau, 16/17. Juli 1948. grenze verschiebt, gilt dies für die sehr viel energiereicheren Ultrastrahlungspartikel, deren Geschwindigkeiten von der des Lichtes ja nur um Prozente und Bruchteile davon verschieden sind, noch lange nicht.

Wir müssen also erwarten, daß deshalb Höhenstrahlungsteilchen aus Raumgebieten empfangen werden, deren Sterne infolge der Doppler-Verschiebung bereits außerhalb der optischen Sichtbarkeitsgrenze liegen; die energiereichen Partikel werden sozusagen aus viel größeren Bereichen der Welt gesammelt als Licht, und dieser Effekt mag wohl in der Lage sein, die oben erwähnte Diskrepanz zu beheben. Ob er quantitativ ausreicht, ist nicht ohne ausführliche kosmologische Untersuchungen $\mathrm{zu}$ entscheiden, auf die wir in dieser Arbeit verzichten wollen.

Es sei jedoch noch auf einen zweiten Gesichtspunkt hingewiesen, der in diesem Zusammenhange von Interesse ist.

Bekanntlich ist die Intensität der kosmischen Strahlung im Mittel zeitlich außerordentlich konstant, wenn man von sekundären terrestrischen Einflüssen absieht. Der beobachtete Sternzeitgang besitzt eine Amplitude, deren Größe nur Bruchteile eines Prozents der Gesamtstrahlung ausmacht.

Dies ließe sich im Rahmen unseres Bildes so verstehen, daß die Wirkung der räumlich ziemlich ungleichförmig verteilten „Quellen“ aus der Milchstraße und deren näherer Umgebung selbst nur von der Größenordnung eines oder einiger Prozente ist, während die mit wachsender Entfernung immer besser isotrop angeordneten Entstehungszentren der Ultrastrahlung den Hauptbeitrag liefern. Für die letzteren ist darum eine Auszeichnung bestimmter kosmischer Gegenden kaum mehr zu erwarten, und dies muß dazu führen, daß die Intensität der Ultrastrahlung aus diesen Bereichen der Welt keine Abhängigkeit von der Sternzeit aufweist.

\section{BESPRECHUNGEN}

Der Bau des Weltalls. Von He in rich Vogt. CESBücherei Bd.16. Curt E. Schwab-Verlag, Stuttgart 1949. $108 \mathrm{~S}$. mit 18 Abb., Preis geb. DM 4.80. „Dies Büchlein will darüber berichten, was der Fachastronom heute über den Bau des Weltalls aussagen kann." So umreißt Verf. im Vorwort das Ziel der kleinen, etwas über 100 Seiten umfassenden
Schrift, in der er ein eindrucksvolles Bild des Universums zeichnet. Das Hauptgewicht liegt dabei auf den elliptischen und Spiralnebeln, die in großer Zahl den Weltraum erfüllen und die größten bisher bekannten Strukturelemente des Kosmos darstellen. Der Erörterung ihrer Formen, Dimensionen, Massen und physikalischen Eigenschaften sowie ihrer schein- 